\title{
The cryoEM structures of immature and mature Zika virus and of mature Zika virus complexed with a human monoclonal antibody
}

Devika Sirohi, Zhenguo Chen, Lei Sun, Thomas Klose, Theodore C. Pierson, Michael G.

Rossmann, Richard J. Kuhn

Vidya Mangala Prasad, Andrew Miller, Thomas Klose, Devika Sirohi, Geeta Buda, Richard J. Kuhn, Michael G. Rossmann

S. Saif Hasan, Andrew Miller, Gopal Sapparapu, Estefania Fernandez, Thomas Klose, Feng Long, Andrey Fokine, Jason C. Porta, Wen Jiang, Michael S. Diamond, James E. Crowe, Jr. Richard J. Kuhn, Michael G. Rossmann

Speaker: Michael G. Rossmann, Dept. of Biological Sciences, Purdue University, West Lafayette, IN 47907, USA

The recent rapid spread of Zika virus and its unexpected linkage to birth defects and an autoimmune-neurological syndrome has generated worldwide concern. Zika virus is a flavivirus like dengue, yellow fever and West Nile viruses. The structure of mature Zika virus is similar to other known flavivirus structures except for the 10 amino acids that surround the Asn154 glycosylation site found in each of the 180 envelope glycoproteins that make up the icosahedral shell. The carbohydrate moiety associated with this residue, recognizable in the cryo-electron microscopy (cryo-EM) electron density, may function as an attachment site of the virus to host cells. This region varies not only among Zika virus strains but also in other flaviviruses and suggests that changes in this region influence virus transmission and disease.

During infection of host cells, virions first assemble into an immature form of the virus. The surface of the immature Zika virus consists of 60 trimeric spikes each consisting of three copies of the precursor membrane (prM) protein and three copies of the envelope (E) glycoprotein. The cryo-EM structure of the immature ZIKV has been determined at a resolution of $9 \AA$. The cryo-EM map of the immature Zika virus is similar to the structures of other immature flaviviruses. The immature zika virus structure shows evidence of an internal capsid structure similar to the internal capsid structure of alphaviruses, but not previously seen in flaviviruses

The cryo-EM structure of an infectious ZIKV in complex with a neutralizing Fab fragment that targets the $\mathrm{E}$ protein has been determined at $6.2 \AA$ resolution. This structure shows that the Fab molecule cross-links monomers within E-glycoprotein dimers as well as between neighboring $\mathrm{E}$ protein dimers, thus preventing the reorganization of E-glycoprotein dimers into fusogenic trimers, required for low $\mathrm{pH}$-triggered fusion with the host endosomal membrane. 